\title{
Colgajo submentoniano: aplicaciones clínicas y variantes en su diseñio
}

\section{Submental flap: clinical applications and variations in the design}

\author{
Mauricio MENDIETA ESPINOSA* ${ }^{*}$, Julio PALACIOS JUÁREZ ${ }^{* \star}$ \\ Armando SIU BERMÚDEZ ${ }^{\star}$, Ricardo FERNÁNDEZ RIERA ${ }^{\star \star *}$
}

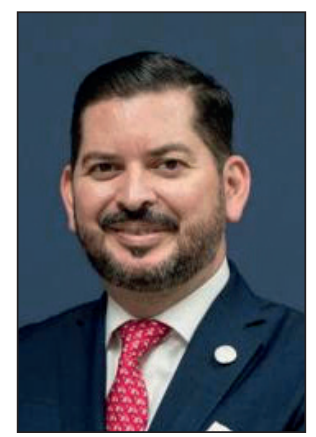

Mendieta Espinosa M.
Resumen

Introducción y objetivo. El colgajo submentoniano ha sido descrito como alternativa para la cobertura de defectos cutáneos en tercio medio e inferior de la cara y como colgajo microquirúrgico para tratamiento del linfedema secundario de extremidades, al incorporar en su diseño nódulos linfáticos vascularizados.

El presente estudio pretende justificar el uso del colgajo submentoniano en sus distintas variantes con la incorporación tradicional de músculo digástrico, perforante de arteria submentoniana, de flujo reverso o microquirúrgico, y con mínima morbilidad del sitio donador.

Material y método. Presentamos una serie de 8 pacientes en los que realizamos un colgajo submentoniano para cobertura de defectos en tercio medio e inferior de cara y de linfedema secundario de extremidad superior. Los colgajos tuvieron dimensiones de 40 a $84 \mathrm{~cm}^{2}$ (media de $62 \mathrm{~cm}^{2}$ ).

Los colgajos fueron pediculados con base en la arteria submentoniana en 4 casos $(50 \%)$, con preservación del músculo digástrico basado en un vaso perforante de la arteria submentoniana en 2 casos, como colgajo submentoniano de flujo reverso en 1 caso, y como colgajo submental linfático vascularizado en 1 caso.

Resultados. Recogimos como complicación una necrosis parcial distal de un $20 \%$ en un colgajo submentoniano de flujo reverso, que fue tratada con cicatrización dirigida a segunda intención. La zona donadora se trató en todos los casos con cierre primario.

Conclusiones. En nuestra opinión, el colgajo submentoniano es una herramienta útil en la reconstrucción de tejidos blandos del tercio medio e inferior de la zona facial, con un grosor, textura y coloración similares, así como una alternativa en el tratamiento del linfedema secundario de la extremidad superior.

Palahras clave Colgajo submentoniano, Reconstrucción cabeza y cuello, Colgajos locales

\section{Nivel de evidencia científica Recibido [esta versión] \\ Aceptado}

$4 c$ Terapéutico

3 mayo/2019
19 noviembre/2019
Background and objective. The submental flap has been previously described as an alternative for the coverage of cutaneous defects or the middle and lower thirds of the face, as well as a vascularized lymph node flap for the treatment of secondary lymphedema of the extremities.

Our goal is to justify its clinical application, and the use of the variants of the components of the flap and design, either with the inclusion of the digastric muscle, submental perforator, reverse flow or microvascular flap, with minimal complications.

Methods. We present a series of 8 patients in which a submental flap was performed to restore cutaneous defects of the middle and lower thirds of the face, and the treatment of secondary lymphedema of the upper extremity. Flaps dimensions were between 40 to $84 \mathrm{~cm}^{2}$ (average of $62 \mathrm{~cm}^{2}$ ).

The flap was designed as submental pedicled flap in 4 cases $(50 \%)$, with preservation of digastric muscle based on a submental perforator in 2 cases, reverse flow submental flap in 1 case, and as a vascularized submental lymph node transfer flap y 1 case.

Results. Complications occurred in 1 of the cases, presented as a partial necrosis of a $30 \%$ of a submental reverse flow flap, which healed by secondary intention. Donor site was closed primarily in all the cases.

Conclusions. In our opinion, the submental flap is a good choice for the coverage of cutaneous defects of the middle and lower face, providing a thickness, texture and color similar to neighboring tissues, as well as an alternative for the treatment of secondary lymphedema of the upper extremity.

Key words Submental flap, Head and neck reconstruction, Local flaps

\section{Level of evidence Received [this version] Accepted}

$4 c$ Therapeutic $3 \mathrm{May} / 2019$

Conflicto de intereses: Los autores declaran no tener ningún interés financiero relacionado con el contenido de este artículo. Financiación: No hubo fuentes externas de financiación para este trabajo. 


\section{Introducción}

En las últimas tres décadas se han logrado grandes avances en la reconstrucción de cabeza y cuello con colgajos libres a pesar de la edad avanzada de los pacientes, la cual no contraindica el procedimiento. Sin embargo, no todos los pacientes se pueden beneficiar de este gran recurso debido a comorbilidades, por lo cual los colgajos pediculados se mantienen como una alternativa viable. Es ahí donde el colgajo submentoniano se presenta como una opción de sencilla ejecución, con anatomía relativamente constante y que se puede realizar en un corto tiempo operatorio.

El colgajo submentoniano fue descrito como una alternativa para la restauración de defectos cutáneos faciales del tercio medio e inferior de la cara, aportando una coloración y textura similares a las de los tejidos faciales, y ya fuera como colgajo regional o libre, con la ventaja de provocar una cicatriz poco perceptible en el sitio donador. ${ }^{(1)}$ Posteriormente, estudios clínicos y experimentales han mostrado que el colgajo submentoniano es versátil en su variante pediculada o libre microquirúrgica y su refinamiento ha permitido una mayor versatilidad en su diseño ${ }^{(2)}$ (Fig. 1).

Como colgajo de cubierta de defectos superficiales, en el sexo masculino tiene la ventaja de transferir unidades pilosas que restituyen áreas del labio superior y de la mejilla que cuentan con esta particularidad,,$^{(3)}$ lo que evita la apariencia en parche que producen los colgajos carentes de unidades pilosas y que hacen más evidente el estigma de enfermedad y de reconstrucción; se logra así un mejor camuflaje de las cicatrices resultantes en base también a lograr la restauración de las unidades estéticas de la cara. ${ }^{(4)}$

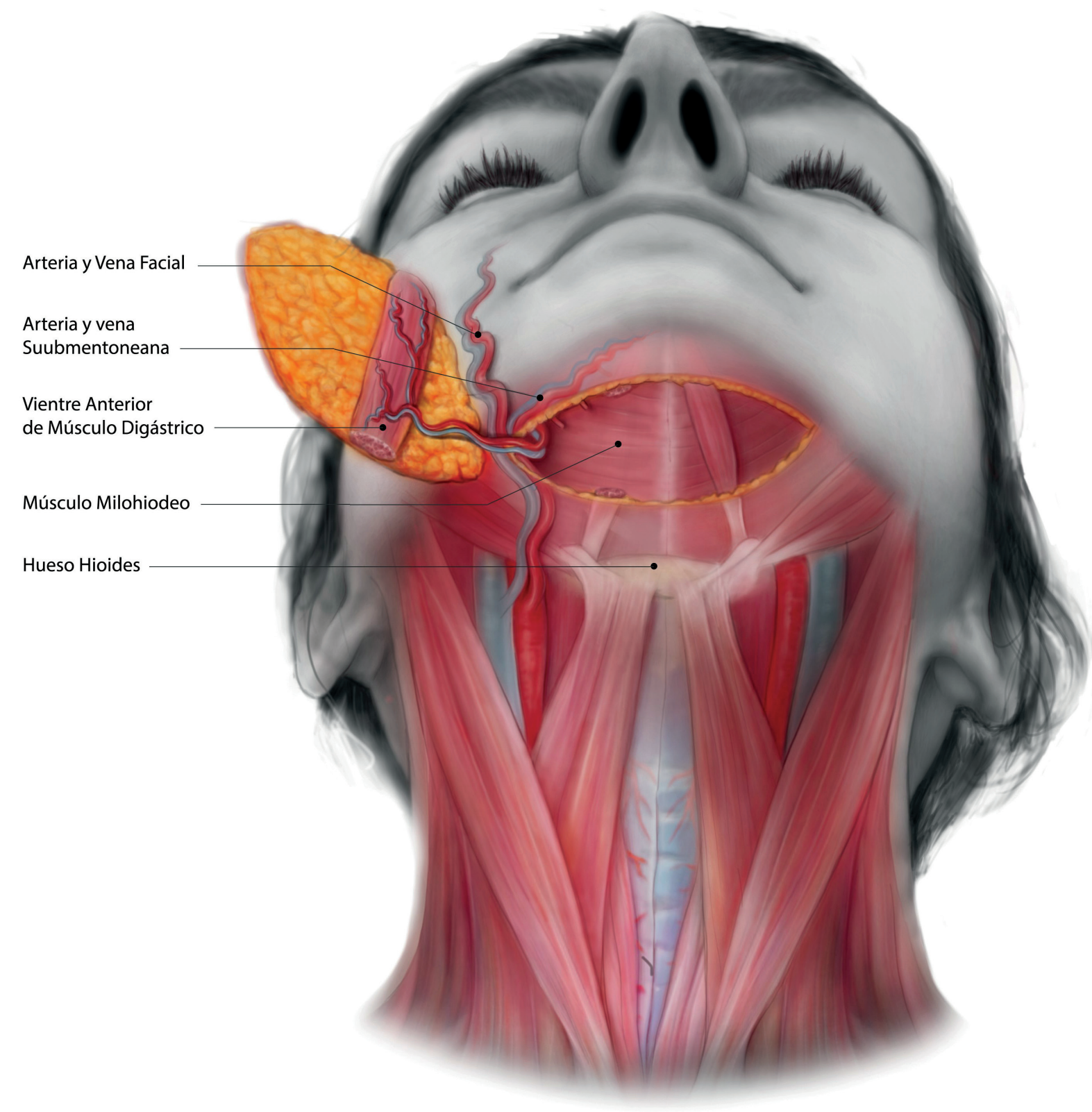

Figura 1. Colgajo submentoniano con estructuras anatómicas de referencia. 
El colgajo submentoniano ha sido descrito también como un colgajo linfático vascularizado para el tratamiento del linfedema, con buenos resultados en los casos de linfedema secundario a mastectomía radical modificada. ${ }^{(5-8)}$

El presente estudio tiene como intención justificar la utilización del colgajo submentoniano como alternativa quirúrgica para la cobertura de defectos cutáneos en la zona facial, ya sea como colgajo perforante o en su variante clásica basada en la arteria submentoniana, y como un recurso microquirúrgico para el tratamiento del linfedema, con mínima morbilidad del sitio donante y bajo índice de complicaciones, todo ello mediante una técnica fácilmente reproducible en distintos medios hospitalarios.

\section{Anatomía vascular de colgajo submentoniano}

La arteria submentoniana es una rama constante, con un diámetro promedio de $2 \mathrm{~mm}$, que emerge a $5-6 \mathrm{~cm}$ del origen de la arteria facial. Transcurre en un surco en la superficie medial de la glándula submandibular, limitado medialmente con el musculo milohioideo y por encima del borde mandibular. La arteria submentoniana brinda 4 vasos colaterales: ramas pequeñas a la glándula submandibular, ramas musculares para el músculo platisma, digástrico y milohioideo, capa adiposa subplastismal y perforantes cutáneas ( 1 a 4), con un vaso perforante atravesando el platisma y dividiéndose en ramas al plano subdérmico que se anastomosan con las ramas contralaterales. Se origina 3 a $15 \mathrm{~mm}$ del borde mandibular y de 4 a $7 \mathrm{~cm}$ del ángulo mandibular. ${ }^{(1,9)}$

La arteria submentoniana termina a nivel del vientre anterior del músculo digástrico, en donde en un $45 \%$ de los casos da una rama a la glandula sublingual y en un $80 \%$ al labio inferior. ${ }^{(10)}$

Las dimensiones del territorio vascular tienen como mínimo $4 \times 5 \mathrm{~cm}$ y hasta un máximo de $15 \times 7 \mathrm{~cm}$.

El drenaje venoso es constante por parte de la arteria submentoniana en la vena facial, con al menos una vena anastomosante entre la vena facial y la yugular externa. ${ }^{(9)}$

\section{Material y método}

Realizamos un estudio retrospectivo, entre diciembre de 2017 y diciembre de 2018, de los colgajos submentonianos realizados por los autores en pacientes con defectos faciales o linfedema secundario de la extremidad superior en el Centro de Cirugía Reconstructiva del Hospital Vivian Pellas (Managua, Nicaragua) y el Hospital Regional de Alta Especialidad de Ixtapaluca (México, México). Revisamos los registros de las historias clínicas de estos pacientes, recolectando datos personales, diag- nóstico, variante de colgajo utilizado, complicaciones y resultados.

\section{Planificación y diseño del colgajo}

Diseñamos el colgajo al menos $1 \mathrm{~cm}$ por debajo del margen de la mandíbula para evitar una cicatriz visible en el sitio donador, con una forma elíptica para lograr un cierre primario más fácil. Con el paciente en una vista de frente y el cuello en posición central, valoramos la laxitud de la piel cervical mediante pellizcamiento para valorar la dimensión vertical del colgajo y garantizar este cierre. Su longitud horizontal está determinada por el límite del ángulo mandibular. Se han reportado dimensiones de hasta $18 \times 8 \mathrm{~cm}$ para el colgajo submentoniano. ${ }^{(11)}$ La disección del pedículo debe permitir que el colgajo sea transpuesto sin tensión. En caso de requerir mayor longitud del pedículo, se realiza un colgajo submentoniano de flujo retrógrado. ${ }^{(12)}$

\section{Técnica Operatoria}

Colocamos al paciente en decúbito dorsal con elevación de la espalda y a nivel de los hombros. Antes de la antisepsia realizamos marcaje preoperatorio con ayuda de un acústico manual CareFusion Pocket-Dop II (Nicolet $\AA$, Middleton, WI, EE.UU.) de 8 Mhz, con angulación del transductor a 45 grados sobre el eje vascular de la arteria facial y submentoniana, así como de las perforantes músculo-cutáneas provenientes del vientre anterior del músculo digástrico. También marcamos la isla cutánea que cubrirá el defecto, a la cual incrementamos $5-10 \mathrm{~mm}$ más del área que deseamos reconstruir.

Si necesitamos un colgajo grueso, generalmente es más sencillo realizar el procedimiento por un abordaje lateral, ya que iniciamos incidiendo piel, tejido subcutáneo y platisma para continuar en ese plano hasta encontrar el vientre anterior del músculo digástrico, que incluiremos para evitar el daño de las perforantes; continuamos la disección sobre el borde mandibular evitando dañar la rama marginal del nervio facial.

Para encontrar fácilmente el trayecto de la arteria y vena submentonianas, disecamos el borde mandibular; primero ubicando el trayecto de la arteria facial en su ascenso y aproximándonos en sentido proximal hasta encontrar su bifurcación que da origen a la arteria submentoniana. Generalmente la encontraremos no exactamente en el borde, sino en el labio ínfero-posterior del cuerpo mandibular. Para evitar el daño de la rama marginal, avulsionamos las fibras del platisma con tijera de tenotomía y vamos protegiéndola con la misma a la vez que disecamos con ayuda de energía monopolar y punta fina Colorado $^{\circledR}$ (Stryker Corporation, EE.UU.). En el abordaje lateral, iniciamos la disección del lado contralateral 


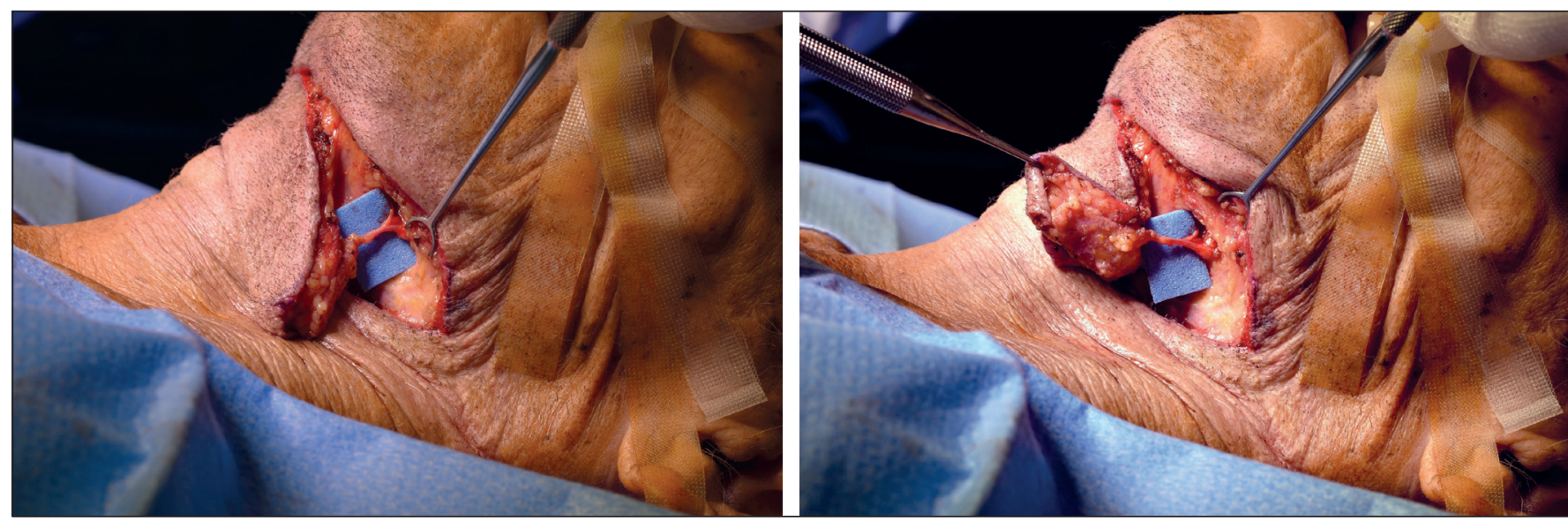

Figura 2. Disección transoperatoria de vaso perforante único con pedículo de $2 \mathrm{~cm}$, con preservación del músculo digástrico.

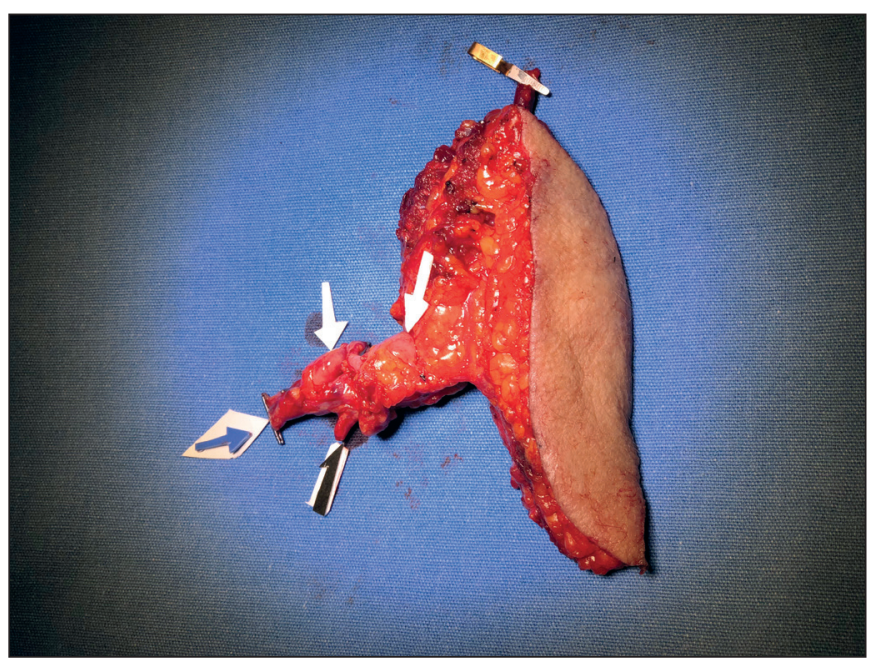

Figura 3. Colgajo submentoniano microquirúrgico (flecha azul=vena; flecha negra=arteria; flechas blancas= nódulos linfáticos, clamp=vena)

al defecto y si visualizamos un vaso perforante adecuado en el punto más cercano al defecto, lo aislamos para ser vaso nutriente del colgajo y rotarlo como un colgajo perforante en hélice, siendo así un colgajo de perforante puro al no incorporar músculo platisma o digástrico ${ }^{(13,14)}$ (Fig. 2).

Preferimos un abordaje inferior cuando queremos obtener un colgajo con linfonodos, ya que encontramos fácilmente el grupo ganglionar entre la glándula y la vena submentoniana o alrededor de ella; sin embargo, se requiere en este caso un mayor cuidado ya que es fácil dañar la rama marginal del nervio facial. Si deseamos realizar un diseño reverso del colgajo, este es el abordaje de elección junto con el abordaje superior, que posee las mayores ventajas para visualización del nacimiento de la arteria, de la rama marginal del facial y los linfonodos (Fig. 3).

Realizamos el cierre del defecto de forma primaria, con sutura directa en 3 planos.

\section{Resultados}

En el periodo de estudio analizado tratamos 8 pacientes en los que realizamos 8 colgajos para la reconstrucción de defectos faciales y tratamiento de linfedema secundario (Tabla I).

La edad de los pacientes estuvo entre los 46 y los 89 años (media 63 años), siendo el sexo masculino el más recuente con 7 casos (88\%) (Fig. 4).

Entre las zonas afectadas que ameritaron cobertura en la cara, recogimos en orden de frecuencia: 5 casos en mejilla, 1 con extensión a la zona paranasal derecha; 1 caso con afección tanto de labio inferior como de mentón y 1 caso en labio inferior. En extremidad superior derecha registramos 1 caso más (Fig. 5). El diagnóstico principal fue carcinoma basocelular en 7 de los casos, y en 1 paciente un linfedema secundario.

Los colgajos tuvieron dimensiones de 40 a 84 $\mathrm{cm}^{2}$ (media de $62 \mathrm{~cm}^{2}$ ) y estuvieron basados como pe-

Tabla II. CPAS: Colgajo perforante arteria submentoniana. CSFR: Colgajo submental de flujo reverso. CAS: Colgajo arteria submentoniana. CSLV: Colgajo submental linfático vascularizado

\begin{tabular}{|c|c|c|c|c|c|c|c|}
\hline Caso & $\begin{array}{l}\text { Edad } \\
\text { años }\end{array}$ & Sexo & Diagnóstico & Localización & Colgajo & $\begin{array}{c}\text { Dimensiones } \\
(\mathrm{cm})\end{array}$ & Complicaciones \\
\hline 1 & 89 & Masc. & Carcinoma basocelular & Mejilla dcha. & CPAS & $12 \times 5$ & No \\
\hline 2 & 46 & Fem. & Linfedema secundario & Extremidad sup. dcha. & CSLV & $8 \times 5$ & No \\
\hline 3 & 56 & Masc. & Carcinoma basocelular & Mentón - Labio inferior & CAS & $12 \times 6$ & No \\
\hline 4 & 84 & Masc. & Carcinoma basocelular & Mejilla - Paranasal dcha. & CSFR & $14 \times 6$ & Necrosis parcial \\
\hline 5 & 54 & Masc. & Carcinoma basocelular & Mejilla dcha. & CAS & $10 \times 5$ & No \\
\hline 6 & 62 & Masc. & Carcinoma basocelular & Mejilla izda. & CPAS & $11 \times 6$ & No \\
\hline 7 & 64 & Masc. & Carcinoma basocelular & Labio inferior & CAS & $12 \times 4$ & No \\
\hline 8 & 72 & Masc. & Carcinoma basocelular & Mejilla dcha. & CAS & $10 \times 6$ & No \\
\hline
\end{tabular}



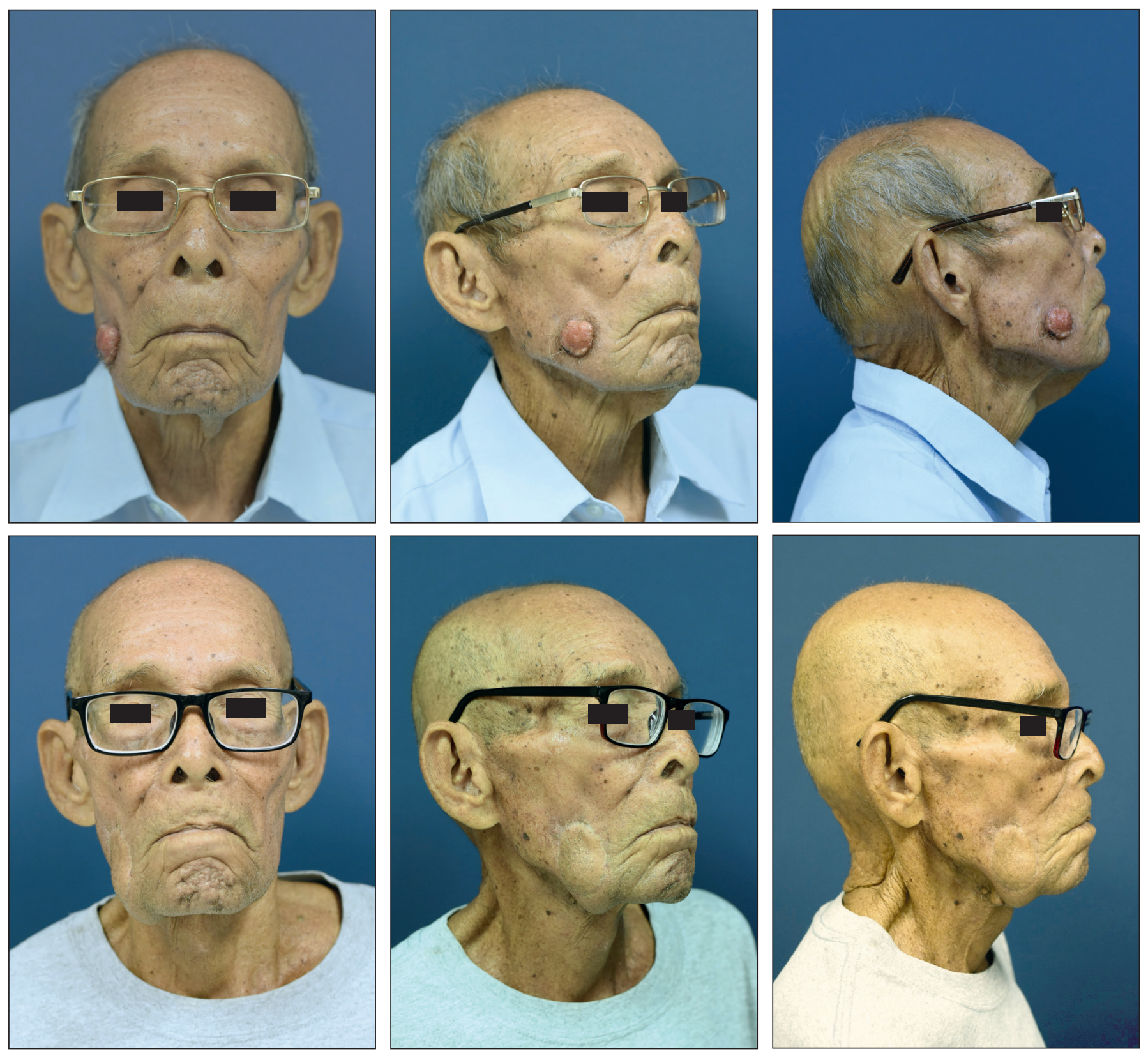

Figura 4. Caso 1. A-C. Preoperatorio de paciente varón de 89 años de edad con diagnóstico de carcinoma basocelular de mejilla derecha. D-F. Postoperatorio a los 9 meses de colgajo submentoniano basado en vaso perforante único preservando el músculo digástrico, traspuesto a mejilla derecha con rotación de 90 grados

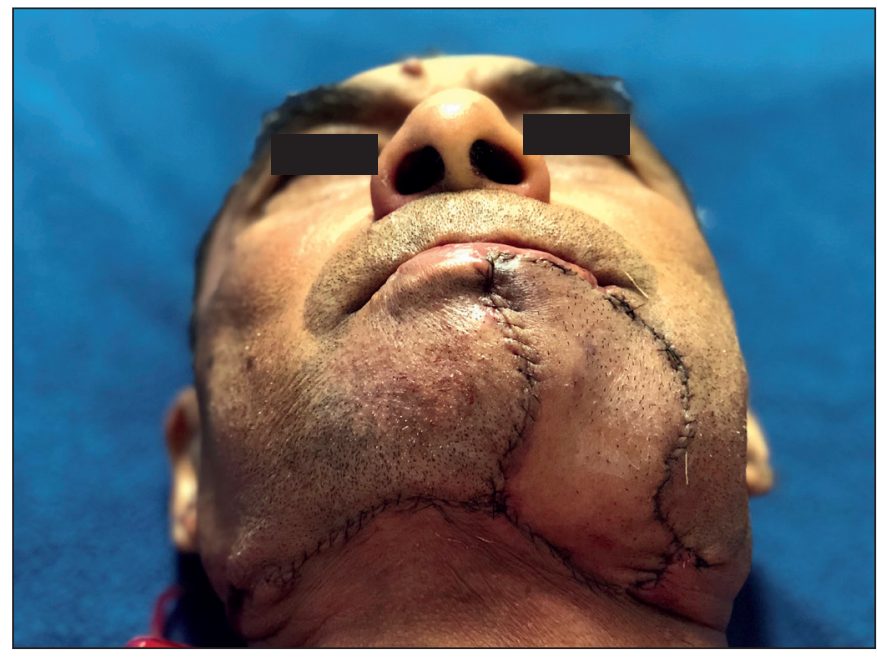

Figura 5. Caso 3. Varón de 56 años de edad con diagnóstico de carcinoma basocelular. Reconstrucción de mentón y labio inferior con colgajo de arteria submentoniana. Imagen del postoperatorio temprano con cicatriz resultante en el pliegue cérvico-mandibular. diculados de la arteria submentoniana en 4 casos $(50 \%)$, en un vaso perforante de la arteria submentoniana en 2 casos, como colgajo submentoniano de flujo reverso en 1 caso, y como colgajo submental linfático vascularizado en 1 caso.

Recogimos una complicación en 1 de los casos: necrosis parcial distal de un $20 \%$ en el caso en que hicimos un colgajo submentoniano de flujo reverso. La tratamos con cicatrización dirigida a segunda intención.

\section{Discusión}

En el presente estudio mostramos como el colgajo submentoniano puede ser utilizado con distintos componentes anatómicos y diseños según las necesidades de la lesión a tratar, preservando el músculo digástrico al 
realizarlo como un colgajo perforante pediculado o en su variante libre para el tratamiento de un linfedema secundario.

La edad juega un papel crítico en la determinación de la isla de colgajo en su dimensión vertical para garantizar el cierre primario del sitio donador. A mayor dad, la piel cervical anterior tenderá a ser más laxa, lo que permitirá un cierre libre de tensión. La edad del grupo de nuestros pacientes facilitó que el colgajo se pudiese tomar y realizar con cierre por primera intención en todos los casos, ya que la media de edad de nuestros pacientes fue de 63 años.

Está demostrado que un área de piel de $10 \times 16 \mathrm{~cm}$ se puede ubicar de forma segura en una sola arteria submentoniana y en su longitud horizontal, el colgajo puede ser diseñado tomando la referencia ósea de un ángulo mandibular al ángulo contralateral, que va a variar según la fisionomía del paciente, lográndose dimensiones de hasta $16 \mathrm{~cm}$, y ser transferido de forma segura con un pedículo vascular unilateral, ya que se han demostrado conexiones vasculares submentonianas en los lados opuestos en un $92 \%$ de los casos. ${ }^{(13)}$ En nuestra serie de casos, la longitud máxima horizontal del área cutánea fue de $14 \mathrm{~cm}$ y la vertical de $6 \mathrm{~cm}$ (media de $62 \mathrm{~cm}^{2}$ ), lo que garantizó la adecuada perfusión de los colgajos realizados (Fig. 6).

Whetzel y Mathes han descrito y caracterizado los vasos perforantes dominantes que recubren las 11 regiones de la cabeza y del cuello, encontrando que las perforan- tes de la rama submentoniana son menos consistentes que las de las ramas de la transversal facial, cigomático-orbitarias, supratroclear, supraorbital y temporal superficial. ${ }^{(15)}$ Se ha llegado a encontrar una perforante confiable en el área submentoniana unilateral en un $87.5 \%$ de los casos, pero con una ubicación bastante variable con respecto al vientre anterior del músculo digástrico. ${ }^{(9)}$ El origen de las perforantes y la relación variable de la arteria submentoniana al vientre anterior del músculo digástrico, han justificado la inclusión del músculo digástrico para garantizar el adecuado suministro vascular del colgajo, motivo por el cual realizamos nuestros colgajos en un $50 \%$ de los casos con la inclusión de este músculo, tal y como se describió originalmente. ${ }^{(1)}$

El colgajo puede ser igualmente basado en el baso perforante situado en la región que cubre el vientre anterior ipsilateral del músculo digástrico, y localizado preoperatoriamente con un doppler acústico manual. Matsui ha llegado a demostrar que un vaso perforante único del colgajo puede minimizar la morbilidad del sitio donador al no incluir músculo y maximizar la movilidad, previendo la perfusión adecuada, lo cual realizamos en un $25 \%$ de los pacientes ya que visualizamos el vaso perforante transoperatoriamente, anterior al vientre anterior del músculo digástrico, lo que previno la inclusión de este músculo en la isla cutánea del colgajo. ${ }^{(16)}$

Al ser todos los pacientes de nuestro estudio de sexo masculino, pudimos incorporar unidades pilosas para re-

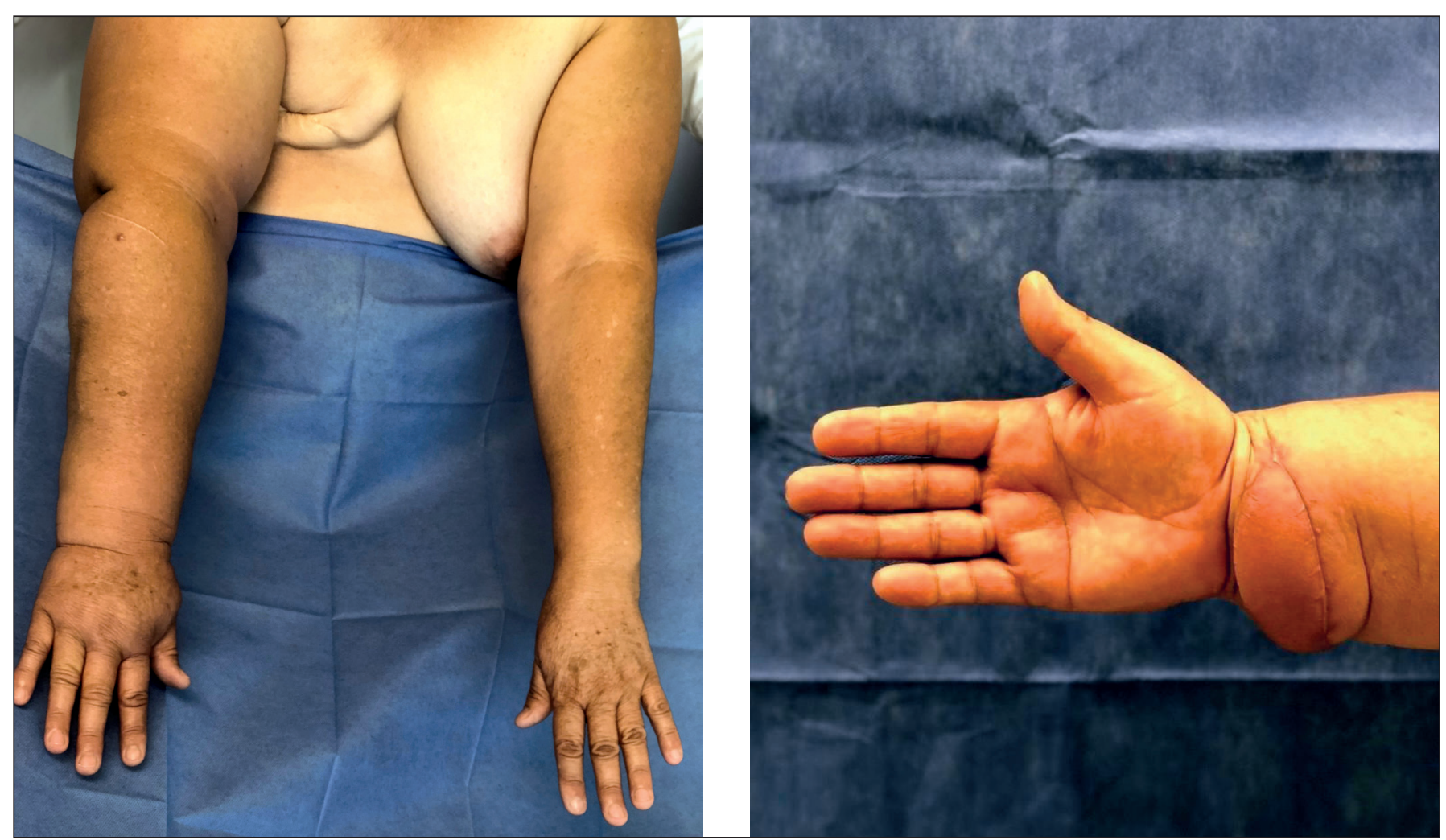

Figura 6. Caso 2. Mujer de 46 años de edad con diagnóstico de linfedema secundario a mastectomía radical modificada por cáncer de mama. A. Vista comparativa de ambas extremidades superiores. B. Colgajo submentoniano a los 6 meses de postoperatorio, con disminución del edema medio palmar de la extremidad superior derecha. 
construir el labio superior y la mejilla restableciendo el área del bigote y la barba, evitando así zonas de alopecia con discontinuidad del vello facial. ${ }^{(9)}$

En caso de requerir un colgajo que se extienda hacia el tercio medio y superior de la cara, se puede utilizar un colgajo de base distal ligando el suministro de sangre anterógrado y basando el colgajo en el suministro de sangre arterial y venosa retrograda a través de la arteria y vena facial distal. ${ }^{(12)}$ Los casos reportados de colgajos submentonianos de flujo inverso advierten sobre la posibilidad de congestión venosa, lo cual ocurrió en el único caso de nuestra serie que realizamos con esta variante y que no solo ameritó la cobertura de la mejilla, sino también de la región paranasal derecha y por tanto precisando un colgajo de mayor tamaño, con una dimensión de $14 \times 6 \mathrm{~cm}$, que sobrepasó la media de los $62 \mathrm{~cm}$ del resto de los colgajos. ${ }^{(12,17)}$

El colgajo submentoniano microquirúrgico utilizado, anastomosado término-lateral a la arteria radial y venas concomitantes con nylon 9-0 para el tratamiento del linfedema secundario a mastectomía, redujo el diámetro de antebrazo de 41.5 a $36.5 \mathrm{~cm}$, de la muñeca de 27 a 21.5 $\mathrm{cm}$, y de la región media palmar de 28 a $22.5 \mathrm{~cm}$ a los 6 meses, mostrando su efectividad con mínima morbilidad del sitio donador, ausencia de linfedema secundario y cicatriz poco perceptible, ${ }^{(7)}$ de manera que, como refieren Scaglioni y col, el colgajo vascularizado submental con linfonodos es efectivo con este método ${ }^{(18)}$ (Fig. 6).

Están recogidas pocas complicaciones de pérdidas parciales o totales de este colgajo en las series descritas por Pistre y col, ${ }^{(11)}$ quienes preservan el músculo digástrico contralateral al defecto en la disección del colgajo, tal y como realizamos en 2 de nuestros casos, utilizando solo el vaso perforante medial al vientre anterior del músculo digástrico, lo que valida al colgajo submentoniano como una gran herramienta en la reconstrucción locorregional de la cara.

El colgajo submentoniano pediculado puede realizarse de forma segura e ir familiarizándose con su anatomía, para posteriormente poder preservar el músculo y realizarlo como un colgajo perforante que sirve como un colgajo pediculado o microquirúrgico, con la adición de linfáticos para el tratamiento de linfedema secundario de las extremidades, secuela en pacientes mastectomizadas.

\section{Conclusiones}

Los objetivos de la reconstrucción de la cabeza y cuello son restaurar la función y apariencia estética del paciente de forma expedita con una morbilidad mínima, lo que hace del colgajo submentoniano el ideal para la cobertura de defectos medianos en los tercios medio e inferior de la cara, al aportar de esta manera un tejido vecino con grosor, textura y coloración similares; sin olvidar que su utilidad con la incorporación de linfáticos vascularizados es una herramienta útil en el tratamiento del linfedema.

Dentro de nuestro estudio, los colgajos submentonianos nos permitieron realizar la cobertura inmediata de los defectos creados como parte del tratamiento del cáncer y preservar el músculo digástrico al realizarlo solo con un vaso perforante como fuente de nutrición, para establecida esta variante de diseño, llevarlo como un colgajo microquirúrgico con la adición de linfonodos.

\section{Dirección del autor}

\author{
Dr. Mauricio Mendieta Espinosa \\ Centro Cirugía Plástica Reconstructiva \\ Hospital Vivian Pellas \\ Carretera Masaya Km 9.5 \\ Managua, Nicaragua
}

Correo electrónico: drmauriciomendieta@gmail.com

\section{Bibliografía}

1. Martin D, Pascal JF, Baudet $\mathbf{J}, \mathbf{y}$ cols. The submental island flap: A new donor site: Anatomy and clinical applications as a free or pedicle flap. Plast Reconstr Surg 1993; 92: 867-873.

2. Shi CL, Wang XC. Reconstruction of lower face defect or deformity with submental artery perforator flaps. Ann Plast Surg 2012;69 (1):41-44.

3. Acosta AA, García Duque O, Baeta BP, Sánchez CO, Menéndez CA, Fernández PJ. Tratamiento local de metástasis cutánea facial de cáncer de colon mediante colgajo submentoniano. Cir plást iberolatinoam 2010; 36: 375-378.

4. Gonzalez-Ulloa M. Restoration of the facial covering by means of selected skin in regional aesthetic units. Br J Plast Surg 1956; 9: 212-221.

5. Cheng MH, Huang JJ, Nguyen DH, et al. A novel approach to the treatment of lower extremity lymphedema by transferring a vascularized submental lymph node flap to the ankle. Gynecol Oncol 2012;126(01):93-98

6. Schaverien MV, Badash I, Patel KM, Selber JC, Cheng MH. Vascularized lympn node transfer for lymphedema. Semin Plast Surg 2018; 32.28-35

7. Ho OA, Lin CY, Pappalardo M, Cheng MH. Comparisons of submental and groin vascularized lymph node flaps transfer for breast cancer-related lymphedema. Plast Reconstr Surg Glob Open 2018; 6: e1923.

8. Aljaaly HA, Fries, CA, Cheng MH. Dorsal wrist placement for vascularized submental lymph node transfer significantly improves breast cancer-realted lymphedema. Plast Rconstr Surg Glob Open 2019; 7: e2149.

9. Hofer SO, Mureau MA. Pedicled perforator flaps in the head and neck. Clin Plastic Surg 2010; 37: 627-640.

10. Faltaous A, Yetman R. The submental artery flap: An anatomic study. Plast Reconstr Surg 1996; 97:56-60.

11. Pistre V, Pelissier P, Martin D y cols. Ten years of experience with the submental flap. Plast Reconstr Surg 2001; 108:15761581 . 
12. Kim JT, Kim SK, Koshima I. An anatomic study and clinical applications of reversed submental artery island flap. Plast Reconstr Surg 2002; 109:2204-2210.

13. Magden O, Edizer M, Tayfur V, Atabey A. Anatomic Study of the vasculature of the submental artery flap. Plast Reconstr Surg 2004; 114: 1719-1723.

14. Ishihara T, Igata $\mathbf{T}$, Masuguchi $\mathbf{S}$, et al. Submental perforator flap: location and number of submental perforating vessels. Scand J Plast Reconstr Surg Hand Surg. 2008;42(3):127-131.

15. Whetzel T, Mathes $\mathbf{S}$. Arterial anatomy of the face: An analysis of vascular territories and perforating cutaneous vessels. Plast Reconstr Surg 1992; 89: 591-603.
16. Matsui A, Lee BT, Winer JH, Laurence RG, Frangioni JV. Submental perforator flap design with a near infrared fluorescence imaging system: The relationship among number of perforators, flap perfusion and venous drainage. Plast Reconstr Surg. 2009; 124: 1098-1104.

17. Yilmaz M, Menderes A, Barutcu Q. Submental artery island flap for reconstruction of the lower and mid face. Ann Plast Surg 1997; 39: 20-35.

18. Scaglioni MF, Arvanitakis M, Chen Y-C, Giovanoli P, ChiaShen Yang J, Chang EI. Comprehensive review of vascularized lymph node transfers for lymphedema: outcomes and complications. Microsurgery 2018; 28: 222-229.

\section{Comentario al artículo “Colgajo submentoniano: aplicaciones clínicas y variantes en su diseño"}

\section{Horacio F. MAYER}

Subjefe del Servicio de Cirugía Plástica del Hospital Italiano de Buenos Aires, Universidad de Buenos Aires, Argentina. Vocal del Capítulo de Cirugía Plástica Oncológica de la FILACP. Director del Comité de Educación de ICOPLAST.

Editor en Jefe de European Journal of Plastic Surgery.

El colgajo submentoniano descrito por primera vez por Martin y col. en 1993 se ha tornado un verdadero caballo de batalla para el cirujano plástico reconstructivo no sólo en reconstrucción cutánea de defectos del tercio medio e inferior de la cara, sino también en reconstrucción por secuela de trauma o postresección oncológica de la orofaringe, nasofaringe y esófago. ${ }^{(1)}$

Cuando es empleado en su modalidad pediculada, provee un amplio arco de rotación, es fácil de levantar $\mathrm{y}$ tiene baja morbilidad asociada del sitio dador. Es realmente una gran alternativa en pacientes ASA III con muy baja tolerancia a extensas microcirugías debido a comorbilidades asociadas. Existe una modalidad bipediculada que, aunque limita la movilidad del mismo, permite optimizar su vascularización al recibir flujo desde ambas arterias faciales.

En aquellos centros donde la microcirugía está disponible por contar con cirujanos plásticos con experiencia microquirúrgica, su variante libre es una gran opción como lo demuestran los autores. Un estudio comparativo sobre el uso del colgajo submentoniano libre en reconstrucción lingual demostró que los tiempos quirúrgicos eran menores respecto a reconstrucciones con otras populares opciones como el colgajo radial y el colgajo anterolateral de muslo (ALT), estando este último también asociado a mayor tiempo de hospitalización. ${ }^{(2)}$

En defectos intraorales, la posibilidad de no incluir músculo realmente otorga un colgajo más fino y modelable. En aquellos defectos de mejilla de espesor total, también es posible diseñar un colgajo con dos pastillas cutáneas para reconstruir tanto el lado mucoso como cutáneo de la misma. Se han utilizado versiones desepitelizadas para aumento de partes blandas en microsomías hemifaciales y versiones expandidas para la cobertura de grandes defectos cutáneos. ${ }^{(1)}$

Respecto a su composición, puede incorporar no sólo piel, celular subcutáneo y músculo digástrico, sino también un fragmento del borde mandibular inferior, transformándose en un colgajo osteomuscular para reconstruir ya sea el maxilar superior o la mandíbula. ${ }^{(3)}$ Más recientemente, la asociación de transferencia de ganglios linfáticos ofrece una alternativa más al arsenal terapéutico del linfedema, como se pone de manifiesto en este artículo.

Los autores deben ser felicitados por los excelentes casos clínicos presentados, los cuales han sido muy bien documentados.

\section{Bibliografía}

1. Rahpeyma A, Khajehahmadi S. Submental artery island flap in intraoral reconstruction: a review. J Craniomaxillofac Surg. 2014;42(6):983-989.

2. Zhao Y1, Xiang JZ, Liu FY. Assessment of life quality of for patients after tongue reconstruction with radial forearm free flap, anterolateral thigh perforator flap or submental island flap. Shanghai Kou Qiang Yi Xue. 2017;26(1):111-114.

3. García-de Marcos JA, Arroyo-Rodríguez S, Rey-Biel J. Submental Osteocutaneous Perforator Flap for Maxillary and Mandibular Reconstruction Following Tumor Resection. J Oral Maxillofac Surg. 2016;74(4):860.e1-9. 


\section{Respuesta al comentario del Dr. Horacio Mayer}

Mauricio MENDIETA ESPINOSA

Agradecemos al Dr. Horacio Mayer por su comentario en referencia a la utilidad y variantes del colgajo submentoniano.

La reconstrucción de cabeza y cuello en defectos secundarios a tratamiento oncológico o trauma, sin duda deben ser llevada a cabo con la prontitud necesaria bajo el principio de restauración lo más cercana posible a la normalidad en apariencia y función y con un tejido similar a la vecindad, para lograr el mejor resultado de las subunidades y proveer calidad de vida con el menor estigma posible. Tal y como se utiliza el colgajo frontal para la reconstrucción nasal, técnica descrita en el 700 AC y llevada, bajo las bases anatómicas y técnicas, a su máximo desarrollo por Burget y Menick, ${ }^{(1)}$ el colgajo submentoniano ha mostrado ser una herramienta útil por su vecindad a los defectos del tercio medio e inferior de la cara, por su anatomía relativamente constante, y por estar en una zona donadora en la que se puede ocultar la cicatriz resultante.

En la reconstrucción de defectos cutáneos faciales, siempre los colgajos locorregionales brindan mejor apariencia que los microvasculares, ya que estos últimos son de zonas distantes, variables en grosor, textura y coloración con respecto a la zona facial, por lo que se hace más evidente su apariencia final en parche. Si bien la longitud del colgajo submentoniano no permite una gran cobertura, como sería la totalidad de una mejilla, siempre bajo el concepto de subunidad puede cubrir defectos menores del $60 \%$; y ante un defecto mayor, siempre será mejor ampliar los márgenes sanos y restituir la unidad en su totalidad. La utilidad de la variante perforante de este colgajo está en preservar al músculo digástrico y contribuir así a la masticación, una acción que ya está de por sí un tanto debilitada en pacientes mayores o en estadios avanzados de enfermedad.

Aun teniendo la Microcirugía como recurso en nuestros centros hospitalarios, la valoración de los pacientes y del área donadora para la reconstrucción es fundamental, ya que el colgajo submentoniano y otros colgajos de perforantes locales, pueden considerarse también como de primera línea, por su facilidad de disección, tiempo operatorio y resultado estético-funcional, sin necesidad de anastomosis bajo microscopio. ${ }^{(2)}$ Es además eficaz, tal y como mencionamos y han documentado otros autores, en el tratamiento del linfedema secundario a cáncer de mama.

En los últimos 30 años, la reconstrucción de cabeza y cuello ha evolucionado en la selección de colgajos y técnicas como resultado de la aplicación y combinación de los principios de reconstrucción de los traumatismos de alta energía, con los conceptos de reconstrucción estética de la nariz, labio y cara, así como con la transferencia libre de tejidos.

\section{Bibliografía}

1. Burget GC, Menick FJ. Nasal reconstruction seekiing a fourth dimensión. Plast Reconstr Surg 1986; 78: 145-157.

2. Moubayed SP, Rahal A, Ayad T. The submental island flap for soft-tissue head and neck reconstruction: Step-by-Step Video description and long term results. Plast Reconstr Surg 2014; 133: 684-686. 
